\title{
Comparison of the Accuracy and Speed of Transient Mobile A/C System Simulation Models
}

\author{
Tibor Kiss and Jason Lustbader \\ National Renewable Energy Laboratory
}

\begin{abstract}
The operation of air conditioning $(A / C)$ systems is a significant contributor to the total amount of fuel used by light-and heavy-duty vehicles. Therefore, continued improvement of the efficiency of these mobile A/C systems is important. Numerical simulation has been used to reduce the system development time and to improve the electronic controls, but numerical models that include highly detailed physics run slower than desired for carrying out vehicle-focused drive cycle-based system optimization. Therefore, faster models are needed even if some accuracy is sacrificed.
\end{abstract}

In this study, a validated model with highly detailed physics, the "Fully-Detailed" model, and two models with different levels of simplification, the "Quasi-Transient" and the "Mapped-Component" models, are compared. The Quasi-Transient model applies some simplifications compared to the Fully-Detailed model to allow faster model execution speeds. The Mapped-Component model is similar to the Quasi-Transient model except instead of detailed flow and heat transfer calculations in the heat exchangers, it uses lookup tables created with the Quasi-Transient model. All three models are set up to represent the same physical A/C system and the same electronic controls. Speed and results of the three model versions are compared for steady state and transient operation. Steady state simulated data are also compared to measured data.

The results show that the Quasi-Transient and Mapped-Component models ran much faster than the Fully-Detailed model, on the order of 10- and 100-fold, respectively. They also adequately approach the results of the Fully-Detailed model for steady-state operation, and for drive cycle-based efficiency predictions

CITATION: Kiss, T. and Lustbader, J., "Comparison of the Accuracy and Speed of Transient Mobile A/C System Simulation Models," SAE Int. J. Passeng. Cars - Mech. Syst. 7(2):2014, doi:10.4271/2014-01-0669.

\section{INTRODUCTION}

When operated, the $\mathrm{A} / \mathrm{C}$ system is the largest auxiliary load on a vehicle. $\mathrm{A} / \mathrm{C}$ loads account for more than $5 \%$ of the fuel used annually for light-duty vehicles in the United States [1]. A/C loads can have a significant impact on hybrid electric vehicle, plug-in hybrid electric vehicle, and electric vehicle performance. Hybrid electric vehicles have $22 \%$ lower fuel economy with the $\mathrm{A} / \mathrm{C}$ on [2]. Mitsubishi reports that the range of the $\mathrm{i}-\mathrm{MiEV}$ can be reduced by more than $45 \%$ on the Japan $10-15$ cycle when the $A / C$ is operating [3]. The advanced powertrain research facility at Argonne National Laboratory has reported an $18 \%$ reduction in range for the Nissan Leaf operating on the UDDS cycle with $35^{\circ} \mathrm{C}$ ambient and 850 [W/ $\mathrm{m}^{2}$ ] solar simulator loading [4]. Increased cooling demands from the battery thermal management system in an electric vehicle may also add additional loads on the A/C system. Air conditioning in heavy-duty vehicles also uses significant fuel for both idle [5] and down-the-road operation. A flexible open-source analysis tool is needed to assess the A/C system impact on advanced vehicles. Industry has expressed a need for both a standalone A/C system model as well as an A/C model that can co-simulate with a vehicle simulator such as Autonomie [].

The A/C system contains complex flow, thermodynamics, and heat transfer. On the refrigerant side, the flow is transient and both compressible and two-phase. Calculating refrigerant properties near the phase transitions can also be computationally difficult. Air flow through the condenser can vary widely depending on vehicle speed and condenser fan speed. The heat transfer in the heat exchangers is a very complex process mainly addressed by complex correlations based on measured data. These correlations sometimes reduce the stability of the code. The effects of humidity and water condensation are difficult to account for, not only in the model but also for the experimental data necessary to calibrate the code. A cabin model is also needed to provide a realistic load on the evaporator. The cabin model must consider all the major pathways of heat transfer into the cabin, including solar and convective loads from the environment, heat from the 
engine compartment, and sensible and latent heat loads in the air stream. Realistic control methods similar to ones actually used in automotive $\mathrm{A} / \mathrm{C}$ systems also have to be implemented. The cycling of the compressor can introduce quick transients that are sometimes difficult to handle by the numerical solver. The simulation model also has to be fast enough for the purpose of evaluating vehicle power performance and/or the design of $A / C$ systems and their controls.

There are numerous challenges in developing a suitable automotive A/C system simulation tool. Some examples of previously developed non-commercial full system-level

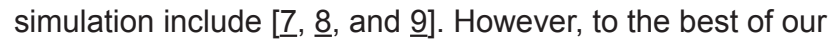
knowledge, none of these or other models have been developed in the widely used dynamic system simulation platform MATLAB/Simulink. Using this platform has several advantages. Autonomie is also built on Simulink, which facilitates integration of the model into Autonomie. MATLAB/ Simulink is widely used in industry, so the standalone, freely available version of the A/C model can be widely distributed. To address these needs, the authors developed a 1-D finite volume $\mathrm{A} / \mathrm{C}$ system simulation model in the Matlab/Simulink environment and compared it to steady state data [10].

This "Fully-Detailed" model provided good agreement with data but ran 10 times slower than real time. It was clear the model was too slow for practical vehicle drive cycle based evaluation. Therefore, the need arose to develop new model versions that provided much faster execution speed while minimizing the loss in accuracy (Figure 1). Drive cycle based studies are especially well suited for such compromise - short transients are less important as long as averaged values for heat transfer rate and compressor power are accurately predicted over long periods of simulation time.

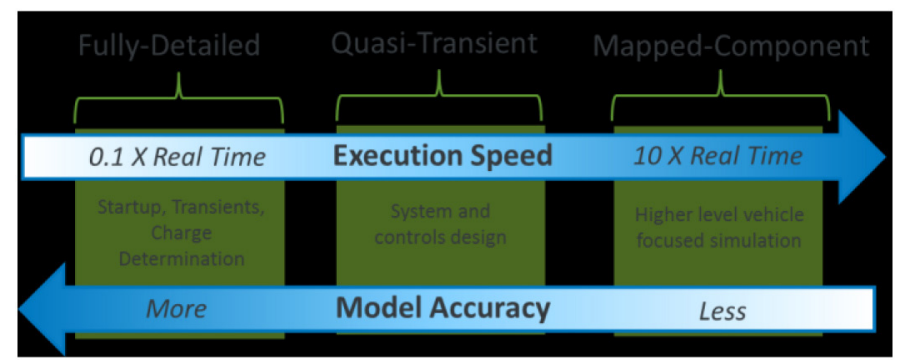

Figure 1. Three model versions to match need with accuracy and speed

To match an appropriate balance of speed and accuracy with the various $A / C$ modeling needs, two additional complementary models were developed, the Quasi-Transient and the MappedComponent model. The user can switch between these models to match the model detail with the current needs of a project. The Quasi-Transient model runs 10 times faster and theoretically matches the steady state conditions of the Fully-Detailed model, but will lose some accuracy during transients. The Mapped-Component model is 100 times faster, but instead of detailed calculations of flow and heat transfer in the heat exchangers, these variables are obtained from multi-dimensional lookup tables.

This paper will briefly summarize the original Fully-Detailed model; for more detail, see reference [10]. The Quasi-Transient and Mapped-Component models will then be described in detail. The results from all three models are then compared to steady state measured data from a light-duty A/C system. Finally, drive cycle averaged system variables from the three models will be compared.

\section{APPROACH}

The new faster Quasi-Transient and the Mapped-Component models were developed from the original Fully-Detailed model. The Quasi-Transient model runs 10 times faster and theoretically matches the steady state conditions of the Fully-Detailed model, but will lose some accuracy during transients. The Mapped-Component model is 100 times faster than the Fully-Detailed model, but instead of detailed calculations of flow and heat transfer in the heat exchangers, these variables are obtained with the help of multi-dimensional lookup tables. The lookup tables used in the MappedComponent models are created with the evaporator and condenser sub-models of the Quasi-Transient model.

Both the Quasi-Transient and the Mapped-Component models incorporate as much of the original Fully-Detailed model as possible. All the descriptions for the cabin, thermostatic expansion valve, and compressor sub-models as well as the described controls algorithm for the Fully-Detailed model apply to these model versions. The heat exchanger structure of the models is also identical between the Fully-Detailed and the Quasi-Transient models. The only difference between the Quasi-Transient and the Fully-Detailed models is that they use different Simulink S-functions (user-defined code) for the zero-dimensional (0-D) volume and one-dimensional (1-D) pipe blocks. In the 1-D pipe simulation blocks, the air-side and the wall temperature calculations are identical for the FullyDetailed and Quasi-Transient version. The MappedComponent model differs from the Quasi-Transient model only in the condenser and the evaporator sub-models where simple multi-dimensional lookup functions are used in place of the detailed heat exchanger models composed of $0-D$ volume and 1-D pipe simulation blocks.

\section{Fully-Detailed Model Summary}

The Fully-Detailed model places an emphasis on predicting transient processes accurately. The model has to be robust for fast transients such as compressor cycling, has to accurately predict refrigerant redistribution after shutdown and after startup, and has to conserve mass accurately during simulations of long test cycles. The more complex and accurate model was also developed first to serve as a benchmark for understanding the error induced through simplifying assumptions that enable faster models. 
A finite volume formulation for calculating the refrigerant flow was chosen because it is well suited for accurate conservation of mass, momentum, and energy. This accuracy is necessary for predicting quick transient processes correctly.

The A/C model consists of two main sub-models, the cooling circuit model and the cabin model. In the cooling circuit model, the larger volumes containing refrigerant (e.g., the accumulator, the receiver/dryer, and the headers of the heat exchangers) are modeled with the 0-D volume simulation block. The refrigerant pipes and the tubes (flat tubes, round tubes, or plates) of the heat exchangers are modeled with various versions of the 1-D pipe simulation block. The refrigerant-side circuit is built up as a network of these 1-D pipe blocks connected to each other with 0-D volume blocks.

In the 0-D volume block, the control volume equations for conservation of mass and internal energy are solved [11]. Because internal energy and mass are the state variables for the simulation, all other refrigerant properties are looked up from two-dimensional tables based on specific internal energy and density.

In the 1-D pipe simulation blocks, on the refrigerant side, equations for conservation of mass, momentum, and energy are solved through a finite volume method. As with the 0-D volume block, all refrigerant properties are obtained from two-dimensional tables based on specific internal energy and density in each finite volume (also called segments). From the applied finite volume method in the 1-D pipe simulation block, it is inherent that the refrigerant flow can take place in both directions. The solution method marches only in time and not in space. The evaluation of heat transfer between the pipe wall and the refrigerant is through local heat transfer coefficient correlations.

The 1-D pipe model simulation allows the option of multiple "parallel channels" on the refrigerant side and complex fin geometry on the external air flow side. It is assumed that the refrigerant flow variables inside the parallel channels are identical. The wall/fin temperatures in the direction of the air flow are assumed to be constant.

For the air flow, there are no conservative terms and the flow is described with purely algebraic equations. Mass flow rate, temperature, and the relative humidity of the incoming wet air for each pipe segment are input variables. The air-side heat transfer equations are solved for each segment of the pipe using the Chang local heat transfer coefficient correlations [12]. The effectiveness-NTU (E-NTU) method [13] is applied on each segment, which ensures that the exit air temperature does not overshoot the wall temperature. Fin efficiency calculations are also incorporated [13].

The effects of humidity and condensation, when present, are accounted for. First, the exit air temperature is calculated from the heat transfer rate as it would be without condensation. If the air temperature drop due to this heat transfer is sufficient to indicate condensation, then an iteration on the exit wet air temperature is invoked. During this iteration the heat transfer to the heat exchanger wall is still assumed to be the same as it would be without condensation. Once the exit wet air temperature is obtained, all other air flow properties can easily be calculated. This same process is applied to all the pipe segments, and the exit air flow conditions are obtained for each pipe segment.

The air-side and refrigerant-side equations can be solved on their own separately because the two sets of equations are not algebraically coupled. The pipe wall temperature, $T_{w}$, appears in both sets of equations, but it is a simulation state variable, which means its value is obtained as a result of an integration step, not from algebraic equations. Therefore, it is available at the beginning of each time step to calculate the heat transfer rates from the air to the pipe wall and from the pipe wall to the refrigerant. The pipe wall temperature couples the equations on the refrigerant and air sides.

It is assumed that the thermal resistance of the wall is zero. In other words, the inner and outer surfaces of the pipe wall are at the same temperature. This is typically a good approximation for compact heat exchangers which use thin walls.

The equation for the wall temperature comes from the conservation of energy, which states that the net heat flux into the wall segment is stored as thermal energy in the wall segment:

$$
\dot{Q}_{a w}-\dot{Q}_{w r}-\frac{d \dot{Q}_{x}}{d x} \cdot \Delta x=C_{p w} * \Delta m_{w} * \frac{d T_{w}}{d t}
$$

where $\dot{Q}_{a w}$ is the heat transfer rate from air to the wall, $\dot{Q}_{w r}$ is the heat transfer rate from the wall to the refrigerant, $\dot{Q}_{x}$ is the heat transfer rate in the pipe wall in the refrigerant flow direction, $C_{p w}$ is the wall material specific heat, and $\Delta m_{w}$ is the mass of the wall segment including all the fins. The $\frac{d \dot{Q}_{x}}{d x} \cdot \Delta x . \Delta x$ term represents the imbalance in conductive heat flow rates from the neighboring wall segments. With this equation, the thermal capacity of the wall has been accounted for. This equation is written for each pipe segment, and the wall temperature is obtained for each pipe segment.

The refrigerant used in the current model is R134a. The effects of the lubricating oil in the refrigerant circuit have not been accounted for.

\section{Heat Exchangers}

Compact heat exchangers are typical in automotive A/C systems and have a general structure that can be described as a number of headers, with "passes" between these headers. Nearly all of the heat transfer takes place in the passes. Using 
conventional terminology, one pass in the condenser consists of a number of round or flat tubes and one pass in the evaporator consists of a number of flat tubes or plates.

Two versions of the 1-D pipe model simulation block described above, with air-side heat transfer according to Chang's model [12], are the basic building blocks for the heat exchangers in the $\mathrm{A} / \mathrm{C}$ system model. To model the tubes of the condenser, a version employing the Dittus-Boelter equation [13] is used on the refrigerant side along the full length of the pass, even across the phase boundaries. In the saturated mix region, the quality weighted averages of the saturated liquid and saturated vapor properties of the refrigerant are used. For the version used to model the plates of the evaporator, on the refrigerant side, the Chen correlation [14] is used for the two-phase region up to $90 \%$ quality, and the Dittus-Boelter correlation with vapor properties is used in the superheat region. A smooth transition between the two is also implemented in the $90 \%-100 \%$ quality range. The Chen correlation was selected because it was originally developed for boiling flow in "conventional" channels as defined by Kandlikar [15], but it was verified by at least two studies that it can be used for minichannel configurations as well $[16,17]$, demonstrating a relatively wide range of applicability. The evaporator plates used in the current study fall into the conventional channel category with a hydraulic diameter of $8.4 \mathrm{~mm}$.

To build a heat exchanger model, each pass/subpass is represented with a 1-D pipe model block that is connected using 0-D volume blocks representing each of the headers. Passes are split up into sub-passes when the air inflow is coming from two different upstream passes. To account for the number of plates in each pass/subpass, the refrigerant mass and enthalpy flow rates have to be multiplied by the number of plates in the pass before being routed into the $0-\mathrm{D}$ volume blocks representing the headers. The total heat transfer rate on the pass/subpass is also the product of the number of plates and the heat transfer rate on just one plate. Therefore, in this approach, all the plates in a given pass are treated as identical in terms of their flow and heat transfer. Figure 2 shows the structure of the evaporator implemented in the model.

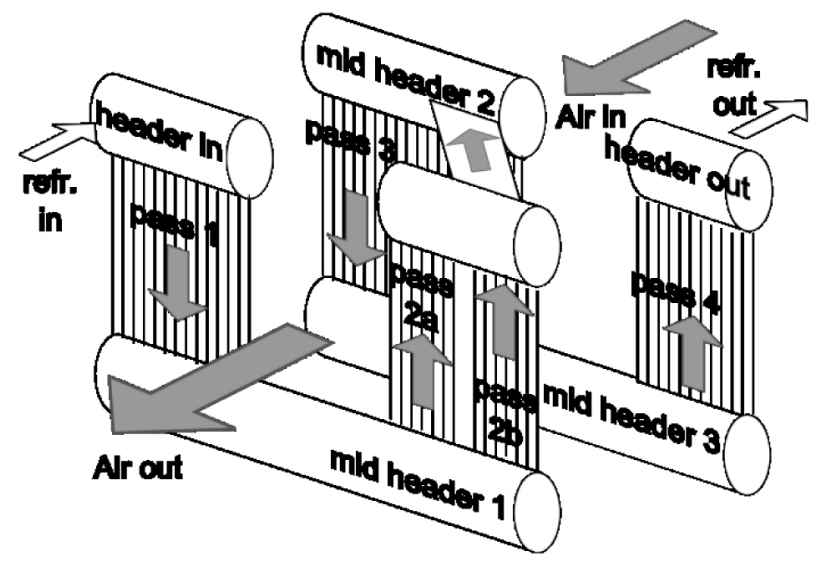

Figure 2. The "exploded view" schematic of the implemented evaporator
Routing the airflow is also relatively easy with the applied modeling method. The segment-wise exit air flow variables from a plate are passed as an output vector from the 1-D pipe simulation block representing the plate. If the air enters another pass in a downstream row, this vector-with proper alignment-is directly fed into the inlet air flow variables input port of the simulation block representing the plate in the downstream row. Alternatively, the exit air flow output vector is mixed with a special simulation block. This option can be used for the last row of the heat exchangers where the airflow from the plate is also exiting the whole heat exchanger.

\section{Thermostatic Expansion Valve}

The expansion device implemented in the model is an externally balanced thermostatic expansion valve, and therefore a receiver/drier is also implemented in the model. The bulb temperature responds to changes in the evaporator exit temperature with a first-order delay. The characteristic time of this response is an input parameter to the model.

This response is the only dynamically modeled detail. Otherwise, the position of the flow restriction device is determined from static force balance. Once the flow area through the flow restriction device of the valve is known, the refrigerant flow rate is calculated from the two-phase orifice flow equations, accounting for choked flow when the pressure drop is high enough.

\section{Compressor}

The compressor is a constant-displacement, variable-speed device. The rotational speed and the displacement per revolution (both input parameters) determine the ideal forwarded volume per second. Actual forwarded volume per second is then obtained with the application of a volumetric efficiency, and the refrigerant mass flow rate is the actual forwarded volume per second multiplied by the upstream density. Refrigerant upstream conditions and downstream pressure are input. The compression process is assumed adiabatic. The downstream enthalpy is calculated with the help of an isentropic efficiency ([18], Eq. 6-62). The compressor shaft power is then the rise in the enthalpy flow rate divided by a mechanical efficiency.

Both the volumetric and the isentropic efficiencies are functions of the compressor speed and the downstream-to-upstream pressure ratios. These tables are inputs to the model. A mechanical drive compressor is used with clutch cycling as explained in the Controls section. The compressor model can easily be changed to that of a variable-displacement device by implementing the appropriate controls.

\section{Cabin Model}

The purpose of the cabin model is to provide a reasonably accurate estimate of the cabin conditions that can serve as the boundary conditions for the cooling circuit model. The cabin air is represented with a zero-dimensional lump-sum air/water 
vapor mix volume. The cabin shell and interior thermal masses are included. Heat transfer between the thermal masses, the cabin air, and the ambient air are accounted for, as well as the solar energy absorption by each thermal mass. The thermal masses and the heat transfer paths that are included in the model are shown in Figure 3.

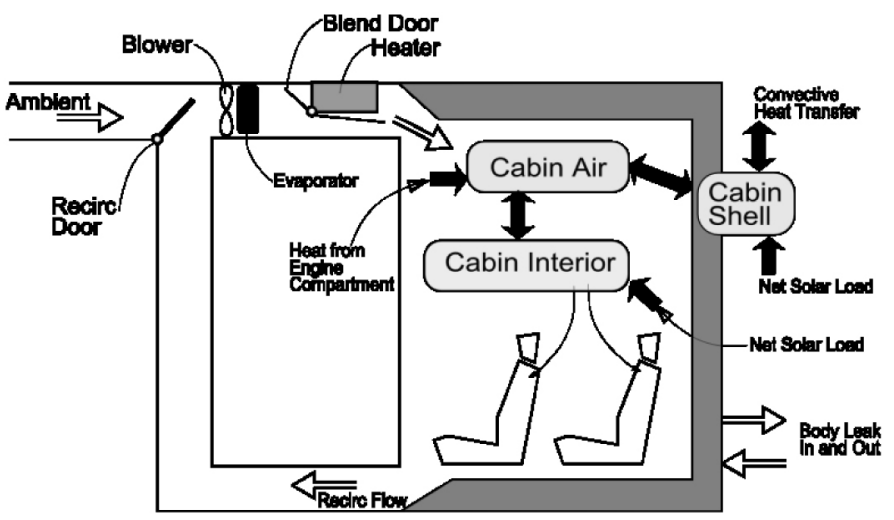

Figure 3. Schematic of the cabin model

The thermal masses are shown in the rounded boxes, and the heat transfer paths are shown with solid arrows. All body air leak flow rates are lumped together and calculated as an adjustable constant times the pressure differential between the cabin and the ambient. The cabin model schematic is shown with the air passages for fresh and recirculated air.

\section{Controls}

Basic electronic controls have been implemented in the model. Shutoff of the compressor due to downstream pressure passing above a high pressure limit, or upstream pressure passing below a low pressure limit is included. The compressor is also cycled on or off depending on whether the cabin temperature is below or above the target temperature and whether the evaporator-out air temperature is below or above a freezing limit temperature. A temperature dead-band is implemented for both of these control variables to reduce the frequency at which the cycling occurs. In addition, limits on how long the compressor has to stay off after each clutch-off cycle is also implemented. The evaporator blower speed is set to one of a number of selectable settings and is not adjusted by the controls algorithm. The cabin air recirculation rate is the portion of air mass flow to the evaporator that is coming from the cabin, the rest being made up with outside air. This rate is set by the controls algorithm in the 5\% - 95\% range based on whether the cabin temperature exceeds the outside temperature or not. Alternatively, the recirculation rate can be set constant over the entire simulation.

These controls are easily modified and additional control algorithms can be added by the user given the block simulation environment of Simulink

\section{The Quasi-Transient Model}

The Quasi-Transient model was created to provide an option for much faster model simulation speed that better facilitates drive cycle-based A/C system studies than the Fully-Detailed model. It is also useful for debugging the Fully-Detailed system model when a new control algorithm or modified components are installed. This model comes at a cost of some accuracy for transient process predictions. The Simulink S-functions that constitute the heart of the simulation model and the Simulink "masks" that improve user communication were created such that any Fully-Detailed model can very quickly be converted into an equivalent Quasi-Transient model or vice versa. This conversion is done by simply swapping out the Fully-Detailed model's 1-D line and 0-D volume Simulink S-functions with their Quasi-Transient equivalents. Some straightforward adjustments to the initialization files also have to be made.

For the Quasi-Transient model, some non-conservation error is allowed to increase the simulation speed. The approach works by pulling the solution towards the steady state solution that belongs to the boundary conditions prevailing at the current time step. Thereby the Quasi-Transient model approximates the solution that would be obtained with a hypothetical quasisteady model that in every time step calculates the steady state conditions. The Fully-Detailed model uses a finite volume formulation in the refrigerant lines with marching in time only whereas the Quasi-Transient model uses a finite volume method with marching in space as well as in time. When all the time derivatives of the flow and other variables are zero, such as in steady state conditions, the solutions to the equations incorporated in the Quasi-Transient model will closely match the solutions of those equations incorporated in the FullyDetailed model.

In the 0-D volumes, a compromise is made to the accuracy of the implemented conservation equations. With this treatment, however, it can be ensured that the volumes in the system model all have similar "stiffnesses" and high stiffness in the volumes with liquid is avoided. This allows for a larger simulation time step resulting in much faster model execution.

The 1-D pipe model used in the Quasi-Transient model has a refrigerant mass flow rate that is constant along the line and is also a simulation state variable. In each time step, the refrigerant pressure drop across the line is compared to the pressure drop obtained from the two attached $0-D$ volumes. A numerical method is applied to continuously adjust the refrigerant mass flow rate in the lines to match the pressure drop in the line to the pressure drop between the attached 0-D volumes better. The refrigerant mass flow rate responds with some delay, but it is tending towards the flow that would develop under the steady state conditions.

The cost of the approach used for the 0-D volume and 1-D pipe models is that the total refrigerant mass in the system is slightly fluctuating and the energy balance is slightly off, especially for 
shorter simulated time periods. For steady state conditions, however, the conservation of mass and energy for each $0-D$ volume and each 1-D pipe in the model is valid.

The equations used for the Quasi-Transient model reflect the goal of approximating the quasi-steady solution; that is, a system response solution that is a series of steady state solutions belonging to the current system boundary conditions. For example, if the system was in a steady state condition and a boundary condition (say cabin temperature) takes a unit step, the model will correctly predict the steady state conditions prior to the step and the new steady state conditions developed after the step, but not the intermediate conditions of the system in between. The equations and method detailed in the following sections will successfully produce this behavior.

There are significant approximations involved in the QuasiTransient model; however, it provides a good balance between accuracy and speed. Ultimately, the utility of this model is determined by how fast it executes and how well it approximates the results of the Fully-Detailed model.

\section{The 0-D Volume Model}

To achieve the goal of the Quasi-Transient model, the concept of 'artificial mass' of the refrigerant is introduced which allows the adjustment of the system 'stiffness'. The conservation equations are written for this artificial mass. The size of the $0-D$ volume is an input parameter and it does not change in time. Mass and enthalpy flow rates into and out of the volume are input variables. The heat transfer rate across the solid boundary of the volume is also an input variable. Conservation of artificial mass is implemented. The time derivative of the artificial mass in the volume is the difference between the sum of incoming and the sum of outgoing mass flow rates.

$$
\frac{d m_{a}}{d t}=\sum_{i} \dot{m}_{i n, i}-\sum_{j} \dot{m}_{o u t, j}
$$

where $m_{a}$ is the artificial mass, $t$ is time, and $\dot{m}_{i n, i}$ and $\dot{m}_{o u t, j}$ are incoming and outgoing mass flow rates, respectively. Conservation of energy is handled similarly with a form of the control volume equation. The size of the volume is constant, which means there is no work on the solid boundary surfaces that would have to be accounted for. Therefore, the time derivative of the total energy in the volume is the sum of incoming enthalpy flow rates minus the sum of outgoing enthalpy flow rates plus heat addition:

$$
\frac{d U}{d t}=\sum_{i} \dot{H}_{i n, i}-\sum_{j} \dot{H}_{o u t, j}+\dot{Q},
$$

where $U$ is the internal energy, $\dot{H}_{\text {in }}$ and $\dot{H}_{\text {out }}$ are the enthalpy flow rates in and out the volume, respectively, and $\dot{Q}$ is the heat transfer rate into the volume through the walls.
Then naturally, $m_{a}$ and $U$ are the simulation state variables. From Eqs. (2) and (3), the integrator can generate the artificial mass and total energy, so these are obtained before any other variables as time is advanced by one time step.

The introduction of the artificial refrigerant mass opens up the possibility to change how pressure and density are connected through the refrigerant material property equations; in fact, this is the reason behind introducing the artificial mass. In this model, the refrigerant in the volume is represented by one single bulk modulus valid for all conditions. With that, the pressure is a function of the artificial density only. The bulk modulus is also proportional to the size of the volume. This ensures that the volumes in the system model all have adjustable and identical 'stiffness'. Equal "stiffness" in all the volumes means that the addition of unit refrigerant mass to any of the 0-D volumes in the system will result in the same pressure rise. With this treatment, high stiffness in the volumes with pure liquid present is avoided. The result is a higher simulation time step allowed and therefore a much faster model execution. Accordingly, the pressure is

$$
p=B \cdot\left(\frac{m_{a} / V}{\rho_{\text {ref }}}-1\right)
$$

where $B$ is the bulk modulus, $V$ is the size of the volume and $\rho_{\text {ref }}$ is a reference density. Note that while $V$ is varying from volume to volume in the model, $B / V$ is the same for each refrigerant volume ( $0-\mathrm{D}$ volume) in the model. $B / V$ is an input parameter as well as the volume, and $B$ is calculated. The lower the value that is given to $B / V$, the "softer" the system will be. By dividing the total enthalpy with the artificial mass, the specific enthalpy in the volume can be obtained as

$$
h=(U+p V) / m_{a}
$$

Temperature is calculated from the pressure and the specific enthalpy, based on the property tables. The "actual" density or just density is also calculated. It is noted that this density is not the artificial density that comes from the artificial mass divided by the volume, but instead, like the temperature, is calculated from the pressure and specific enthalpy using the accurate property tables. This is done so that the true refrigerant mass in the system, the one that is consistent with the pressures and enthalpies all over the system, can be evaluated.

It should be noted that Eqs. (2) and (3) reduce to the accurate equations for conservation of mass and energy when applied to steady state conditions. In addition, the model in every time step-if the boundary conditions are frozen-would start converging to the steady state solution belonging to those frozen boundary conditions.

To this latter point the following observations can be made. If the sum of incoming mass flow rates is greater than the sum of outgoing mass flow rates, the artificial mass will increase, and 
therefore the pressure in the volume will increase. This pressure rise will reduce the incoming mass flow rates and will increase the outgoing mass flow rates. In the end, the system will change in the direction of the incoming and outgoing mass flow rates to become more equal, that is, towards a steady state solution. A similar statement can be made for the enthalpy exchange, at least for the case when the mass flow rates already reached steady state. Then, if the sum of the enthalpy inflow rates plus the heat transfer rate is greater than the sum of the enthalpy outflow rates, the internal energy in the volume will rise, which will increase the outgoing enthalpy flow rates but will not affect the incoming enthalpy flow rates. The result is again that the system variables are changed in the direction of the steady state solution.

Therefore, Eqs.(2) and (3) reduce to the rigorous mass and energy conservation equations in steady state conditions and they will move the system towards the correct steady state conditions from any transient conditions.

\section{The 1-D Pipe Model}

These equations were also developed with the goal of approximating the quasi-steady solution. In such a solution, the refrigerant mass flow rate is constant along the length of the pipe at any time, although it varies in time. As was mentioned earlier, on the air side and for the wall temperature calculations, this 1-D pipe model is identical to the 1-D pipe model of the Fully-Detailed model.

A finite volume formulation is used again to determine the lengthwise distribution of flow parameters, and this requires splitting up the line into finite volumes or "segments." With the refrigerant mass flow rate fixed along the length of the pipe, the finite volume equations can be applied with a marching scheme in the direction of the flow. For each segment and in each time step, the flow variables on the outlet boundary of a segment can be calculated from the flow variables on the inlet boundary of the segment and the wall temperature of the segment. Assume for now that the magnitude and direction of the refrigerant mass flow rate is known as we start calculating the distributed flow parameters. Then the inlet boundary conditions of the first segment are those prevailing in the $0-D$ volume attached on the upstream side of the 1-D pipe block. No slip velocity is assumed, and the flow is assumed to be homogeneous.

First the pressure on the exit boundary of the segment, $p_{\text {out }}$, is calculated using the Darcy-Weisbach equation ([11], Eq. 5.8.7).

$$
p_{\text {out }}=p_{\text {in }}-1 / 2 f \frac{L}{D_{H}} \rho_{\text {in }} v_{\text {in }}^{2}
$$

where $p_{i n}, \rho_{i n}$, and $v_{i n}$ are the pressure, the density, and the velocity on the inlet boundary of the segment; $L$ is the length of the segment; and $D_{h}$ is the hydraulic diameter. $v_{n}$ is the constant mass flow rate, $\dot{m}$, divided by the inlet boundary density, $\rho_{i n}$. The wall friction coefficient, $f$, is obtained from the Hagen-Poisseuille equation ([11], Eq. 5.10.12) for laminar flow and from a modified version of the Colebrook equation ([11], Eq. 5.10.13) for turbulent flow, always using the inlet boundary flow variables. When two-phase flow is present on the inlet boundary of the segment, viscosity is a quality averaged value of the saturated vapor and saturated liquid viscosities at the inlet boundary temperature. In accordance with the above, no void fraction based correlation was implemented for the pressure drop calculation. It is noted that the pressure drop calculation used the same basic equations as the FullyDetailed model.

Next the local heat transfer coefficient is calculated with essentially the same correlations as for the Fully-Detailed model. When two-phase flow prevails on the inlet boundary, refrigerant material properties are the quality weighted average of the saturated liquid and saturated vapor properties. The default for calculating the local heat transfer coefficient is the Dittus-Boelter correlation [13]. For only the two-phase boiling flow in the evaporator plates, the Chen correlation [14] is used.

If single-phase flow prevails on the inlet boundary, the E-NTU method [13] as applied for constant wall temperature is used in the refrigerant flow direction to obtain the exit temperature. This ensures the exit temperature from the segment does not overshoot the wall temperature and that no restriction needs to be placed on the length of the segment from a stability point of view:

$$
T_{\text {out }}=T_{\text {in }}-\left(T_{\text {in }}-T_{w}\right) \cdot\left(1-\exp \frac{\alpha A}{\dot{m} C_{p}}\right)
$$

where $T_{\text {in }}$ is temperature on the inlet boundary, $T_{w}$ is the segment wall temperature, $A$ is the heat transfer area (segment length times inner channel perimeter) $\alpha$ is the heat transfer coefficient and $C_{p}$ is the constant pressure specific heat. It is possible, however, that the flow is single phase on the inlet boundary, but turns into two-phase flow within the segment. For example, the location at which condensation starts in the condenser does not have to line up with a segment boundary. Therefore, a check has to be carried out using the exit temperature predicted by Eq. (7). For the condenser example, it is checked whether $T_{\text {out }}>T_{\text {sat }}\left(p_{\text {out }}\right)$. If yes, then there is no phase region change, the $T_{\text {out }}$ calculated in Eq. (7) stands, and the heat exchange rate from the refrigerant to the wall can be calculated as

$$
\dot{Q}=C_{p} \cdot \dot{m} \cdot\left(T_{\text {in }}-T_{\text {out }}\right)
$$

If, on the other hand, $T_{\text {out }}<T_{\text {sat }}\left(p_{\text {out }}\right)$, the refrigerant is moving from superheated vapor into the saturated mix region at some location within the segment. In this case, the distance from the inlet boundary, $y$ at which $T_{\text {out }}=T_{\text {sat }}\left(p_{\text {out }}\right)$, is determined using 
the E-NTU equation, Eq. (7), but substituting $A=y \cdot w$ ( $w$ is the internal flow path perimeter) and solving for $y$. Then the heat transfer rate in the single-phase section of the segment is

$$
\dot{Q}_{1}=\dot{m} \cdot\left(h_{\text {in }}-h_{\text {sat }, v}\left(p_{\text {out }}\right)\right)
$$

where is $h_{\text {in }}$ the enthalpy on the inlet boundary, and $h_{\text {sat' } v}$ is the saturated vapor enthalpy. The heat transfer rate in the twophase region part of the segment is calculated as

$$
\dot{Q}_{2}=A \cdot \frac{L-y}{L} \cdot \alpha \cdot\left(T_{\text {sat }}\left(p_{\text {out }}\right)-T_{w}\right)
$$

The total heat transfer in the segment is

$$
\dot{Q}=\dot{Q}_{1}+\dot{Q}_{2}
$$

Once the heat transfer rate is determined either with Eq. (8) or Eq. (11), the specific enthalpy on the outlet boundary can be calculated with

$$
h_{\text {out }}=\left(\dot{H}_{\text {in }}-\dot{Q}\right) / \dot{m}
$$

where $\dot{H}_{\text {in }}$ is the enthalpy flow rate through the inlet boundary of the segment. From $h_{\text {out }}$ and $p_{\text {out }}$ all other refrigerant properties can be calculated on the outlet boundary.

If there is saturated mix on the inlet boundary, then the temperature change in the segment is likely minimal, and a different method has to be used. The segment exit pressure, $p_{\text {out }}$, is again calculated with Eq. (6). The temperature of refrigerant at the exit is that of the saturated mix temperature at the segment exit pressure, it is not coming from the heat transfer rate. A constant temperature difference along the segment between the wall and the refrigerant can be assumed and the heat transfer rate from refrigerant to the wall can be calculated without the E-NTU method simply as

$$
\dot{Q}=A \cdot \alpha \cdot\left(T_{i n}-T_{w}\right) .
$$

The enthalpy outflow rate is

$$
\dot{H}_{\text {out }}=\dot{H}_{\text {in }}-\dot{Q}
$$

and the specific enthalpy on the outflow boundary is

$$
h_{\text {out }}=\dot{H}_{\text {out }} / \dot{m} \text {. }
$$

Now there is a possibility that the refrigerant goes from saturated liquid / vapor mix on the inlet boundary to single phase flow somewhere within the segment. This can happen for example, in the condenser when the refrigerant enters the subcooled liquid region. The following is for that specific case, but similar equations can be used when the evaporator refrigerant goes from the saturated vapor / liquid mix region to the superheated vapor region.

To check the presence of crossing such a phase boundary, it needs to be evaluated whether $h_{\text {out }}<h_{\text {sat }}$, $\left(p_{\text {out }}\right)$, that is, the exit enthalpy is smaller than the saturated liquid enthalpy at the exit pressure. If the answer is no, the previous equations stand. If the answer is yes, the refrigerant enters the subcooled region somewhere within the segment. Once again, the distance of the location at which the phase change is complete, $y$, is determined. The heat transfer needed to condense all vapor is

$$
\dot{Q}_{1}=\left(h_{i n}-h_{s a t, l}\right) \cdot \dot{m} .
$$

Then $y$ can be calculated from the following equation

$$
\dot{Q}_{1}=\frac{y}{L} \cdot A \cdot \alpha \cdot\left(T_{w}-T_{i n}\right)
$$

Next, the E-NTU method, Eq. (7), can be used for the last $(L-y)$ long part of the segment to determine the segment out temperature $T_{\text {out }}$ by substituting $(L-y) \cdot w$ for the heat transfer area and $T_{\text {sat, }}\left(p_{\text {out }}\right)$ for $T_{\text {in }}$. Then Eq. (8) can be used again with substituting $T_{\text {sat, }}\left(p_{\text {out }}\right)$ for $T_{\text {in }}$ to determine $\dot{Q}_{2}$, which is now the heat transfer to the wall in the subcooled liquid part of the segment. The total heat transfer is the sum of $\dot{Q}_{1}$ and $\dot{Q}_{2}$, and Eqs. (14) and (15) can be used again to determine the exit specific enthalpy.

Finally, from the already calculated exit pressure, $p_{\text {out }}$, and the specific enthalpy on the exit, $h_{\text {out }}$, all refrigerant material properties can be found using the property tables based on $p$ and $h$.

It is also theoretically possible that within one segment the flow goes from superheated vapor to subcooled liquid in the condenser when the compressor is cycled off and mass flow rate quickly drops to near zero. Such case is also treated in the model with similar methodology, which involves splitting the segment into three pieces. The details of this process are not given here.

This completes the explanation of how the outlet boundary flow variables of a pipe segment are calculated from the inlet boundary flow parameters and wall temperature of that segment, for all possible phase change cases. The obtained outlet boundary flow variables now can serve as the inlet boundary flow variables for the next segment. This method is applied to all segments in the pipe, one after another, so the pipe flow is solved in what can be called a "marching" scheme from the upstream boundary to the downstream boundary of the pipe. At the end of this process, a pressure on the outlet boundary of the last pipe segment is obtained. This pressure 
can be compared to the pressure in the 0-D volume connected to the pipe on the downstream boundary of the pipe. Ideally they match within a small error, which would mean the refrigerant mass flow rate used in the calculations is acceptable as that for the steady state conditions. However, in general, the two will differ significantly. One approach could be to iterate on the refrigerant mass flow rate until the two pressures do match. This was tried, but it actually turned out to be very time consuming, as the marching flow calculations have to be repeated many times over. The time penalty was so high that the Quasi-Transient model showed no improvement in speed relative to the Fully-Detailed model.

Therefore, a different approach was used, in which the marching had to be completed only once per pipe per time step. Instead of determining the steady state flow following from the upstream and downstream 0-D volume conditions in each time step, the pipe flow solution is just moved closer to this steady state solution in each time step. In each time step, the refrigerant pressure at the exit from the line - calculated with just one execution of the marching scheme - is compared to the pressure in the $0-D$ volume attached downstream. Based on the result of the comparison, a rate of change of the refrigerant mass flow rate in the pipe is calculated that allows the next time step to have a better match between these pressures. Because the refrigerant mass flow rate is calculated by integrating this rate of change, the refrigerant mass flow rate is now a simulation state variable and therefore available at the beginning of each time step. A graphical explanation is shown in Figure 4. The actual equation applied to calculate the derivative of the mass flow rate is

$$
\frac{d \dot{m}}{d t}=C \cdot \frac{p_{d, c a l c}-p_{d}}{p_{u}-p_{d}} \cdot \dot{m},
$$

where $\dot{m}$ is the refrigerant mass flow rate, $p_{u}$ and $p_{d}$ are the pressures in the upstream and downstream connected 0-D volume blocks, respectively, $p_{d \text {, calc }}$ is the 1-D pipe downstream boundary pressure calculated in the current time step with one sweep of marching, $C$ and is an input parameter that determines the strength of the pull towards the steady state solution.

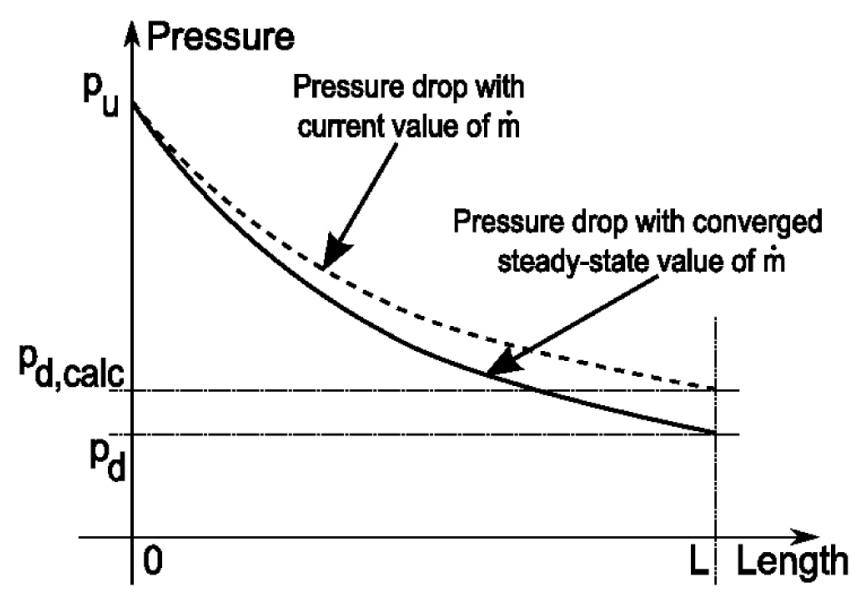

Figure 4. Refrigerant mass flow rate adjustment in Quasi-Transient 1-D pipe simulation block (Eq. (18))
Depending on the value of the factor $C$, the result is a stronger or weaker pull of the mass flow rate and all other flow variables toward the steady state solution prescribed by the attached $0-D$ volumes. Proper setting of the input parameter $C$ is determined after comparing the results of the Quasi-Transient model to the results of the Fully-Detailed model.

\section{Treatment of Mass Conservation}

Note that the "real" refrigerant mass in the 0-D volumes and $1-D$ pipes is an output, and there is nothing implemented in the model with the explained methodology that preserves this mass over time. In a real system, of course, refrigerant mass is preserved, apart from some leakage that usually plays out over a very long time. The system does behave somewhat differently with different amounts of refrigerant charge. Therefore, a fluctuating total refrigerant mass in the system gives little hope to match the behavior of a real system.

The way this issue was addressed in the model was by the addition/removal of "artificial" refrigerant mass based on the instantaneous total 'real' refrigerant mass compared to a target or "charge" refrigerant mass. This can be done because in each 0-D volume and 1-D pipe at each time step, the 'real' refrigerant mass is calculated from the volume, the pressure and the specific enthalpy, and these masses are summed up to provide a simulated refrigerant total mass. A refrigerant removal rate linear with the error between this simulated refrigerant total mass and the target refrigerant mass in each time step can then be applied. Negative error in this context means a need for refrigerant addition. Although somewhat arbitrary, it was chosen that mass is added/removed at a single point in the system, through the receiver/drier. With this implemented, depending on the magnitude of the mass removal rate coefficient, the calculated refrigerant mass could be kept within about $10 \%$ of the target.

\section{The Mapped-Component Model}

Compared to the Quasi-Transient model, the MappedComponent model represents another step towards increased model execution speed at an additional cost of reduced accuracy. The Mapped-Component model is created from the Quasi-Transient model by replacing the condenser and the evaporator sub-models with simplified sub-models that use lookup tables. Otherwise, the two models are identical. The lookup tables were generated with standalone models of the condenser and the evaporator taken out of the Quasi-Transient model.

For the condenser, the refrigerant mass flow rate, the air-torefrigerant heat exchange rate, and the refrigerant mass in the condenser are each obtained from a separate five-dimensional lookup table based on upstream refrigerant pressure, upstream refrigerant specific enthalpy, refrigerant pressure drop, air mass flow rate, and air inlet temperature. For the evaporator, the lookup tables also have to incorporate the relative humidity of the incoming air for acceptable accuracy. Therefore, if the evaporator had been treated similarly to the condenser, the 
lookup tables would be six-dimensional. Adding a sixth dimension to the lookup tables greatly increases the time needed to generate them. The hysteresis due to the evaporator wall thermal mass is also important when the compressor starts cycling for evaporator freeze prevention. To solve these issues, the lookup tables for the evaporator were split into refrigerant-side and air-side tables coupled through the wall temperature. Furthermore, instead of heat exchange rate, effectiveness was looked up on the refrigerant side. Using the effectiveness proved to be more robust to prevent false results in case the conditions were outside the domain of the mapping. For the four-dimensional refrigerant-side tables, the refrigerant mass flow rate, the refrigerant mass in the evaporator, and the wall-to-refrigerant heat transfer effectiveness are looked up from the upstream refrigerant pressure, upstream refrigerant quality, the refrigerant pressure drop, and the temperature differential between the inlet refrigerant flow and the wall. For the air side, the air-to-wall heat transfer rate is looked up from the air mass flow rate, air-in temperature, air-in relative humidity, and the wall temperature. Creating these four 4-dimensional maps is much more time efficient than creating three maps each with six dimensions. With this splitting approach, the time needed to create the evaporator maps was reduced from 86 to 4 hours. Figure 5 shows the input/output variables for the condenser performance lookup tables, and Figure 6 shows the input/ output variables for the evaporator performance lookup tables.

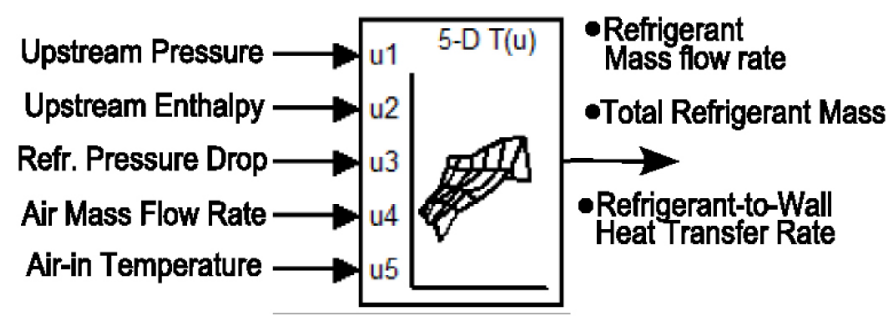

Figure 5. Condenser performance lookup tables
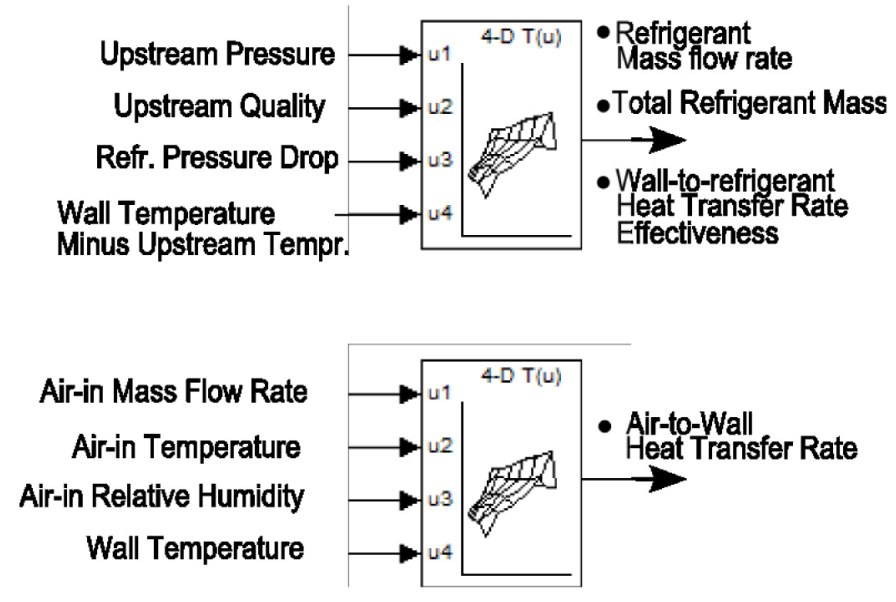

Figure 6. Evaporator performance lookup tables
The lookup tables were generated with standalone models of the condenser and the evaporator taken out from the QuasiTransient model. For each point in the lookup tables, the condenser and evaporator models were run until steady state conditions were reached. The heat transfer rates, the refrigerant mass flow rates, and the refrigerant total masses were recorded and placed into large arrays that the Simulink lookup table blocks of the Mapped-Component model could read. (The refrigerant-side evaporator heat transfer rates first were processed into effectiveness numbers). This entire process was automated. The necessary maps can be created with the automated process on an Intel ${ }^{\circledR} \mathrm{i} 7-3520 \mathrm{M} 2.9 \mathrm{GHz}$ CPU 64-bit personal computer with $4 \mathrm{~GB}$ of RAM in a matter of 10 to 12 hours.

The lookup tables are used in the following way: refrigerant-out enthalpy flow rate is the sum of the refrigerant-in enthalpy flow rate and the heat transfer rate to refrigerant, both for the condenser and the evaporator. The heat transfer rate in the evaporator on the refrigerant side is calculated from the effectiveness. Air-out temperature on the evaporator is calculated the same way as a segment air-out temperature is calculated in the Fully-Detailed model, accounting for condensation of water on the metal surfaces, if present. Because there are no segments, the iteration on air-out temperature has to be carried out only once. Also, there is no need for mixing the segment-out air flows. The refrigerant mass in the evaporator and in the condenser obtained from the lookup tables are added to the refrigerant masses in the rest of the system to obtain the total system refrigerant mass. Then, a similar adjustment of the refrigerant total mass is applied as in the Quasi-Transient model. The refrigerant mass adjustment is important; without it, a good match between the data simulated with the Mapped-Component model and the steady state measured data shown in the next section was not successfully achieved.

In the evaporator sub-model, where the wall temperature is a simulation model state variable, the equation for the wall temperature is the same as in the Fully-Detailed model for a segment wall temperature, Eq. (1). Note that here the $\dot{Q}_{a w}$ and $\dot{Q}_{w r}$ terms are coming from the lookup tables, and there is no spatial distribution of wall temperature. One temperature describes the wall and Eq. (1) is only used once. This also means there is no conductive heat transfer in the walls of the evaporator in the flow direction as the entire wall is at the same temperature.

The assumption of constant wall temperature in space may bring in the most significant error. It is a relatively good assumption in the saturated liquid-vapor mix regions of the evaporator, but it represents more deviation from reality in the 
single-phase superheat region where the refrigerant temperature is rapidly rising, and the wall temperature is also rising as a result.

\section{RESULTS}

\section{Verification with Data}

Calibration and verification of the three models was completed using 22 steady state test bench points from a light-duty vehicle A/C system provided by Halla Visteon. These data covered a wide range of operating conditions. Because the heat exchanger air inflow parameters were known and they were constant in time, the cabin model was essentially deactivated by setting very large thermal masses.

The data set used for verification included pressures and temperatures along the entire refrigerant circuit, properties of the upstream and downstream air streams on the heat exchangers, and the compressor speed and mass flow rate data. More details on the data and its implementation for the calibration and verification study can be found in [10].

All 22 measurement points in the data set were simulated with all three models, and some calibration was done on the heat transfer correlation coefficients for the Fully-Detailed model only. The Quasi-Transient and Mapped-Component models both used the same heat transfer and pressure drop correlation coefficients as the Fully-Detailed model.

In Figures $7 \mathrm{a}$ and $\underline{7 b}$, the thermodynamic cycles for measurement points 4 and 6, respectively, are shown on the pressure-enthalpy space for all three models and for the measurement. These two points are representative of the general quality of the match and represent two points with significantly different boundary conditions. Good match between the simulation and the measurement can be seen.

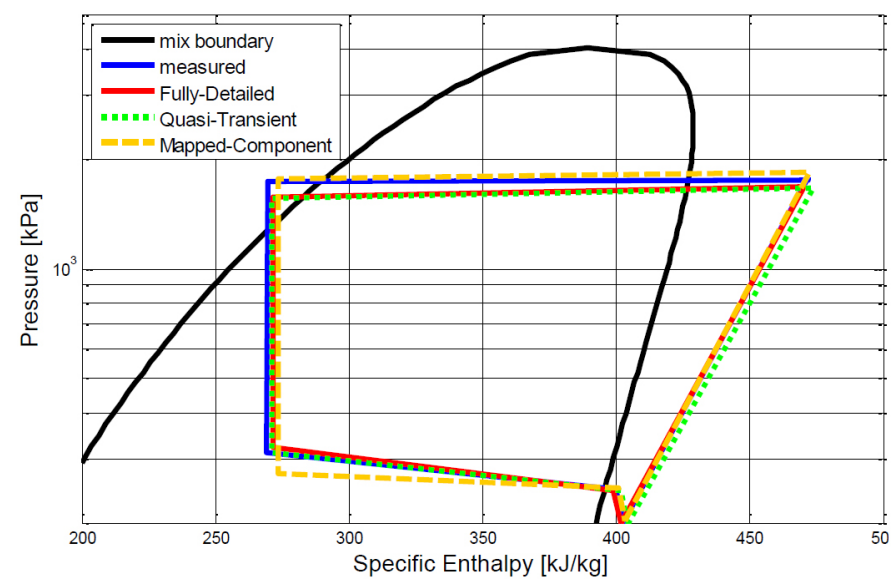

Figure 7a. Thermodynamic cycle for Test Point 4 in the p-h space

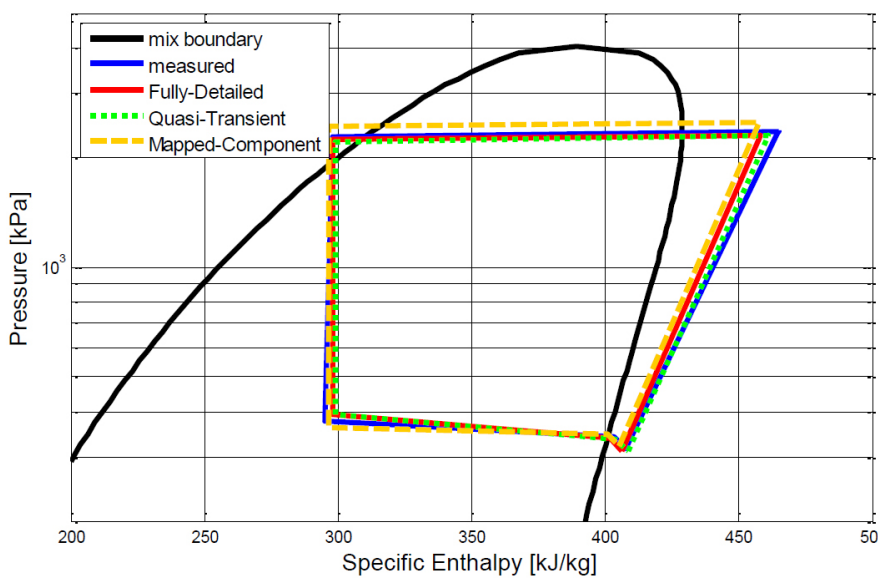

Figure $7 \mathrm{~b}$. Thermodynamic cycle for Test Point 6 in the $p$-h space

\section{Component Level Verification}

The refrigerant flow rate, the heat transfer rate on the condenser, the heat transfer rate on the evaporator, and the evaporator-out air temperature were also generated from the full cycle results. The measured and simulated data for these four variables for all three model versions are included in $\underline{\text { Figures } 8 \mathrm{a}}, \underline{8 \mathrm{~b}}, \underline{\mathrm{8c}}$, and $\underline{8 \mathrm{~d}}$, respectively.

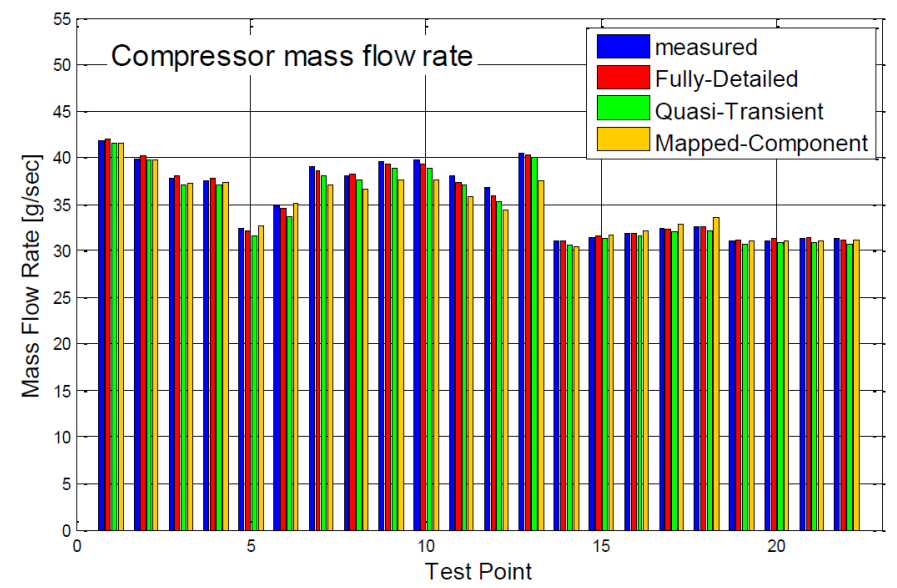

Figure 8a. Predicted and measured compressor mass flow rate

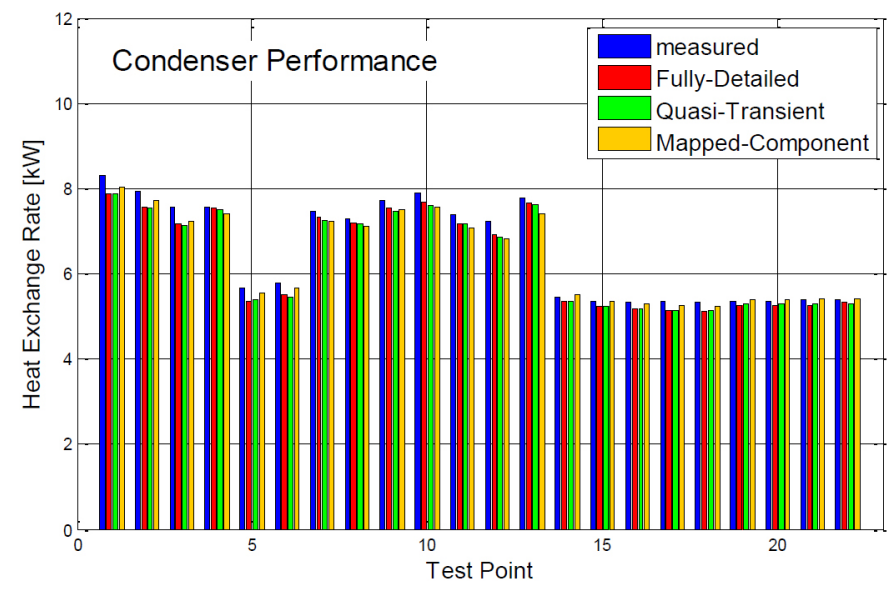

Figure 8b. Predicted and measured condenser heat exchange rate 


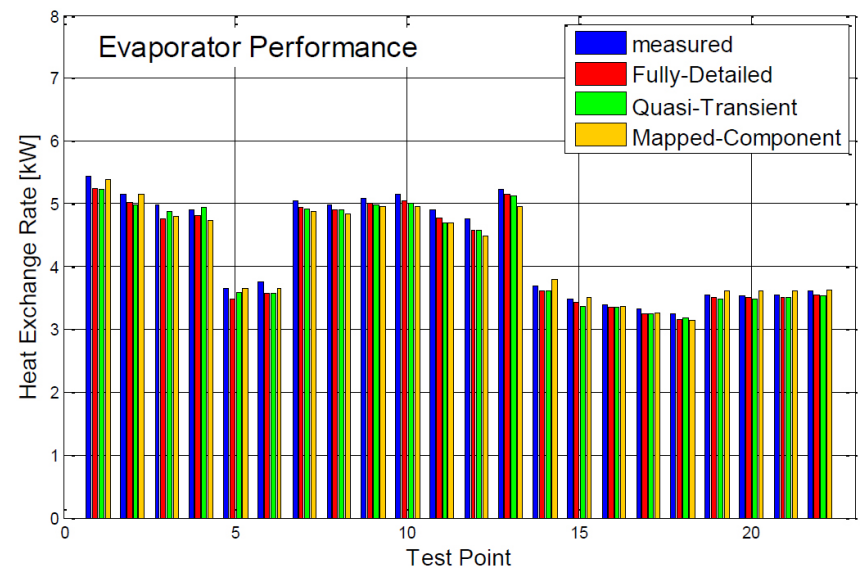

Figure 8c. Predicted and measured evaporator heat exchange rate

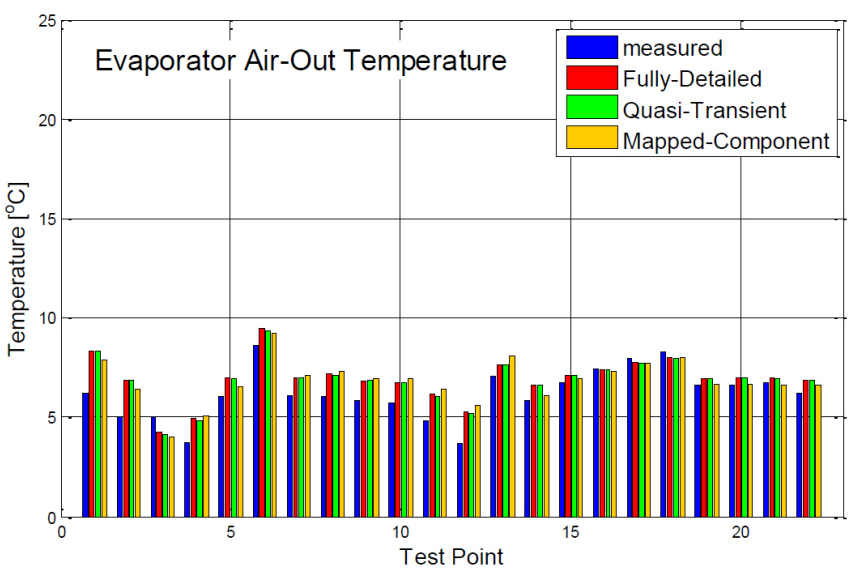

Figure 8d. Predicted and measured evaporator air-out temperature

Figures $8 \mathrm{a}, \underline{8 \mathrm{~b}}, \underline{\mathrm{8c}}$, and $\underline{8 \mathrm{~d}}$ show that a good match between the measured and the simulated data was achieved. As expected, the best match is for the Fully-Detailed model, then the Quasi-Transient, then the Mapped-Component model. The average errors for all three models for all four system variables, that is, for refrigerant mass flow rate, evaporator heat exchange rate, condenser heat exchange rate and evaporator air-out temperature are shown in Figure 9. All are expressed in percent except for the evaporator-out air temperature for which the error is expressed in degrees Celsius.

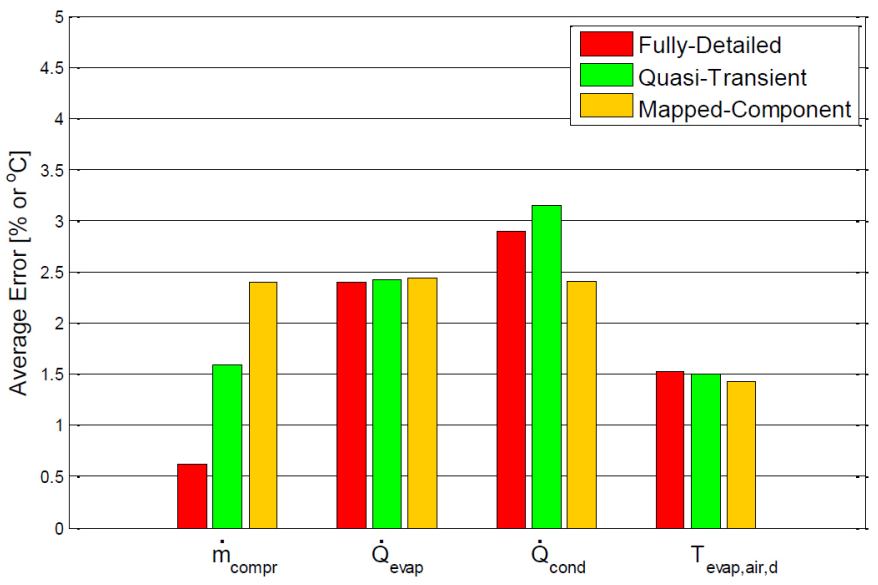

Figure 9. Average prediction errors with the 3 model versions

\section{Comparative Transient Cycle Predictions}

Because no measured data for transient processes were available, results obtained with the Quasi-Transient and Mapped-Component models were compared against results obtained with the Fully-Detailed model.
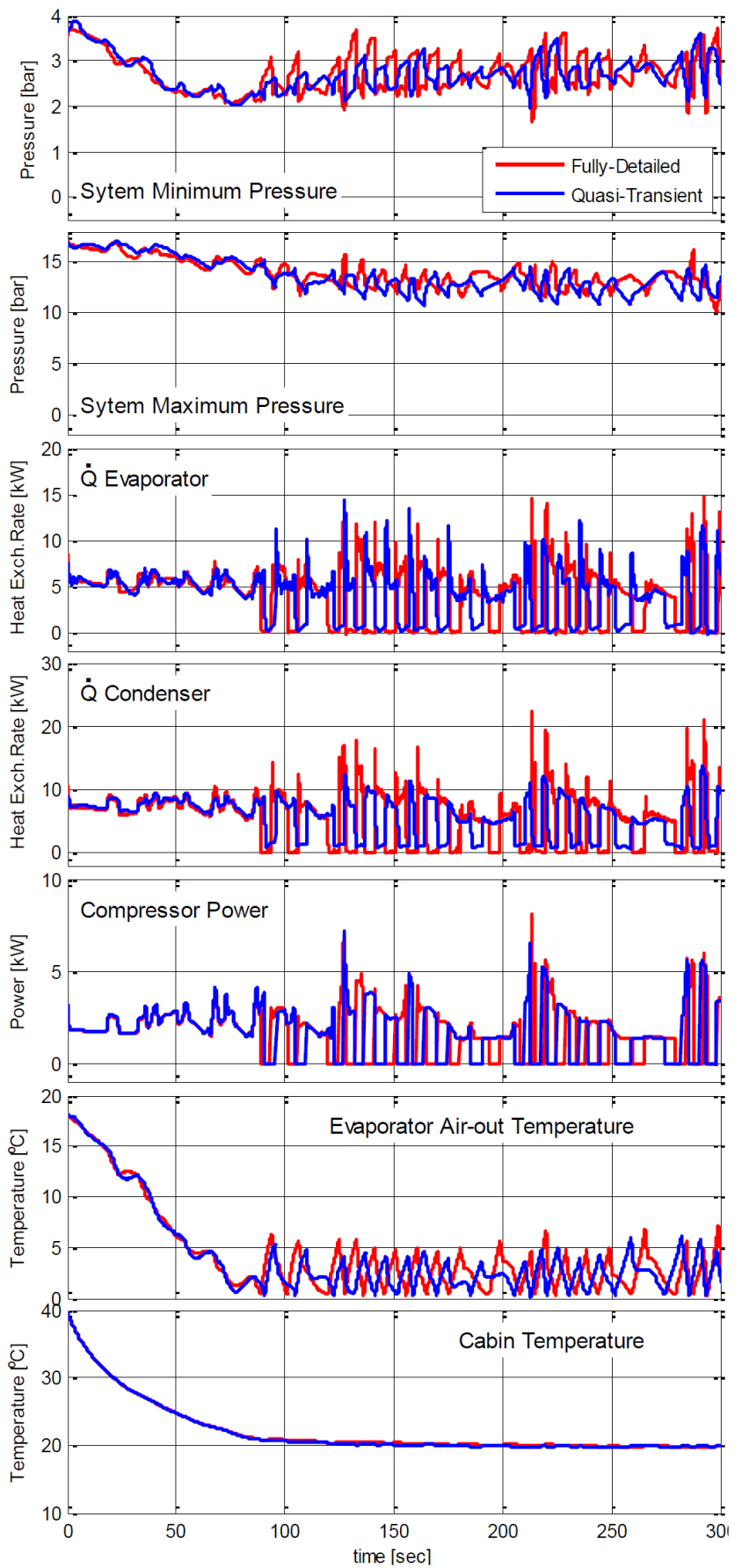

Figure 10. Quasi-Transient model predictions vs. Fully-Detailed model predictions, first $300 \mathrm{sec}$ of SC03 cycle

The SC03 cycle was simulated with all three model versions. The models were set up for the same exact A/C system, the same ambient conditions, and the same cabin initial conditions. The results of the Quasi-Transient model as compared to the Fully-Detailed model for the first 300 seconds of the SC03 cycle are shown in Figure 10. Similar plots comparing the 
results of the Mapped-Component model to the results of the Fully-Detailed model for the first 300 seconds of the $\mathrm{SC} 03$ cycle are shown in Figure 11.

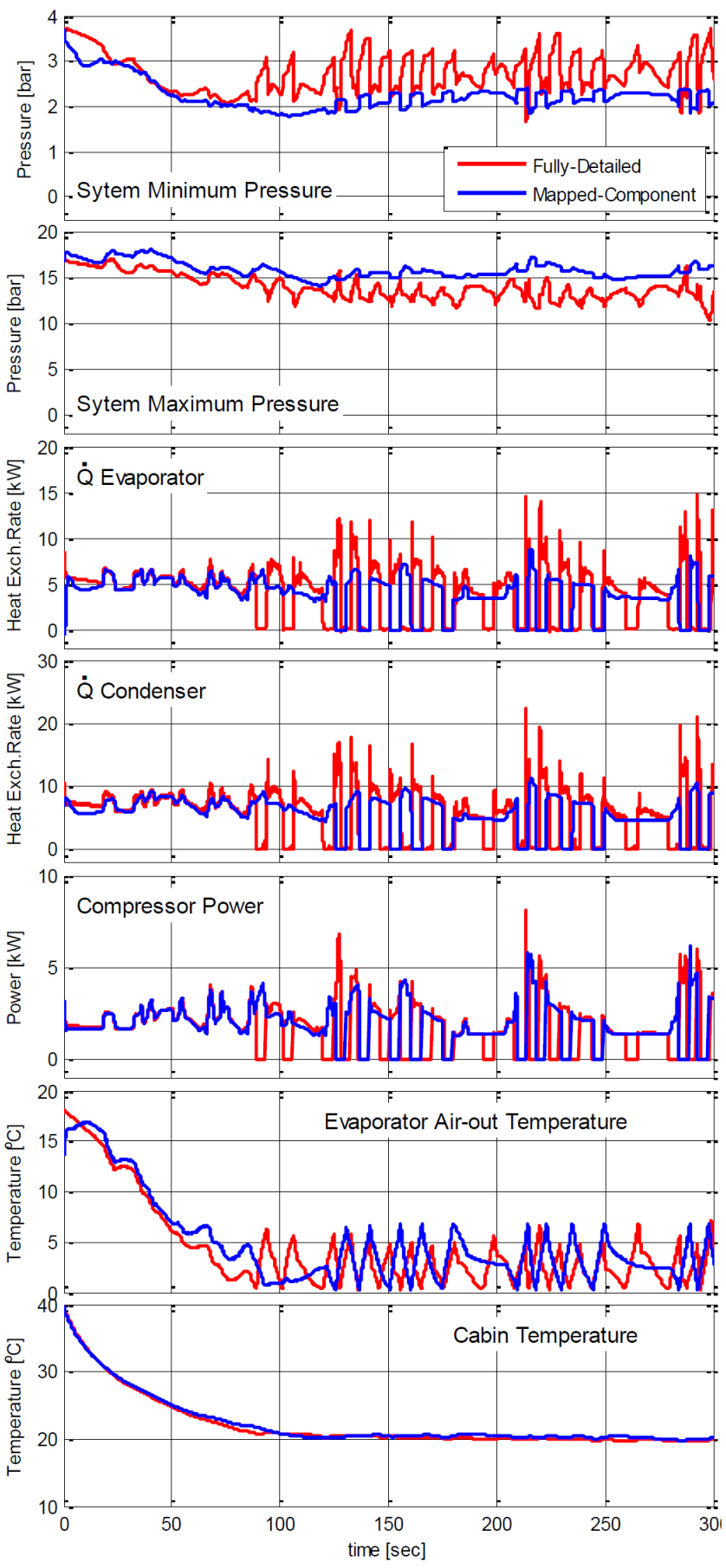

Figure 11. Mapped-Component model predictions vs. Fully-Detailed model predictions, first $300 \mathrm{sec}$ of SC03 cycle

The less accurate, but much faster Quasi-Transient model results match the results of the Fully-Detailed model well. It is notable that the cycling due to evaporator freezing prevention starts at around the same time for the Fully-Detailed and the Quasi-Transient models. Then, due to slight differences in predictions, the start and end points of the cycling periods drift away. After some time, the cycling periods may be far off, yet such discrepancy does not mean there is a large difference in the accuracy of the predictions in general. The discrepancy between the Fully-Detailed and the Mapped-Component model is much larger; even the timing of the first cycling is significantly off.

\section{Drive Cycle Averaged Results}

For vehicle-focused analysis, agreement of the models over a drive cycle is important. Of particular interest are the average compressor power and the average heat transfer rates on the heat exchangers. These data are shown in Figure 12. Over the full SC03 drive cycle, the results with the Quasi-Transient model were within $1.5 \%$ of the results of the Fully-Detailed model for evaporator and condenser heat transfer and compressor power. The Mapped-Component model did not match as well. Although the evaporator and condenser heat transfer rates were within $1.1 \%$ and $4.0 \%$ of the Fully-Detailed models', respectively, the compressor power was $18 \%$ higher than for the Fully-Detailed model. The main reason for this larger difference was an underprediction of the evaporator heat transfer rate by the Mapped-Component model, which resulted in a higher duty-cycle of the compressor.

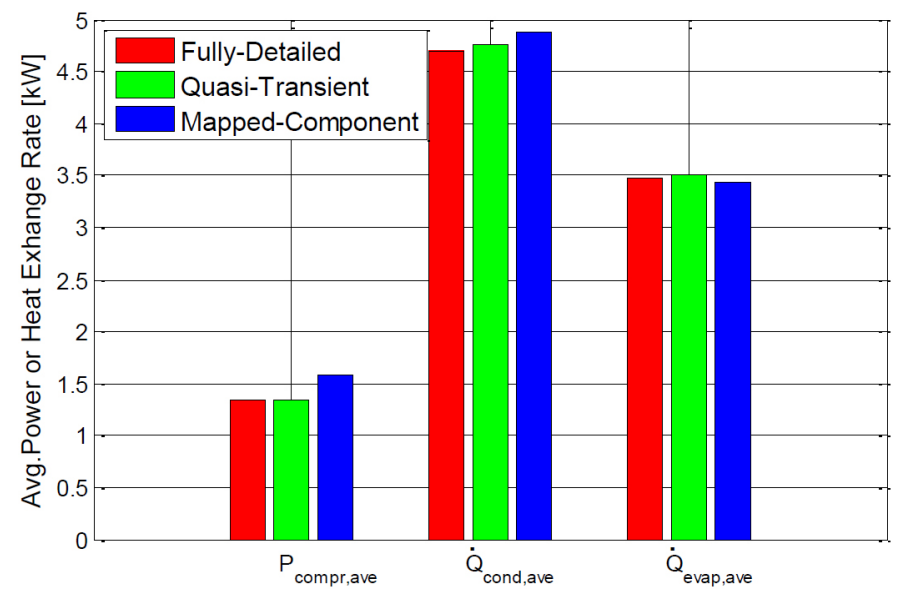

Figure 12. Comparison of drive cycle averaged performance predictions by the three model versions

The drive cycle averaged results also provide an opportunity to evaluate the conservativeness of the models. It was shown previously [10] that the Fully-Detailed model conserves mass extremely well, within $0.001 \%$ error or less. The QuasiTransient and the Mapped-Component models both have a fluctuating refrigerant mass. The fluctuation is mitigated by the addition/removal of some "artificial" mass as explained earlier. It is of interest how close these models keep the actual refrigerant mass to the target refrigerant mass. For the simulation runs shown in Figures 10 and 11 , the refrigerant mass root mean square errors were $6.18 \%$ and $9.31 \%$ for the Quasi-Transient and the Mapped-Component models, respectively. 
Similar analysis can be done for the conservation of energy. The compressor power that applies to this discussion is the power that actually goes into raising the enthalpy of the refrigerant, that is

$$
P_{\text {compr }}=\left(h_{\text {compr,in }}-h_{\text {compr,out }}\right) \cdot q_{m, \text { compr }}
$$

(Note the compressor powers in Figures 10, 11 and 12 incorporate the compressor mechanical efficiency). In steady state conditions, the $1^{\text {st }}$ law of thermodynamics applied for the system states

$$
\dot{Q}_{\text {cond,out }}-\dot{Q}_{\text {evap,in }}=P_{\text {compr }}
$$

if all heat transfer is assumed to take place in the heat exchangers. For transient processes, the terms in Eq. (20) have to be integrated over a time period, and the change in energy stored in the refrigerant also has to be incorporated to be exact. For long periods of simulation, however, the change in energy stored in the refrigerant becomes negligible. Therefore, the energy conservation of the models will be sufficient if the approximation

$$
\int_{t 1}^{t 2} \dot{Q}_{\text {cond,out }} d t-\int_{t 1}^{t 2} \dot{Q}_{\text {evap }, \text { in }} d t \cong \int_{t 1}^{t 2} P_{\text {compr }} d t
$$

holds with good accuracy. The error between the two sides of Eq. (21) for the Fully-Detailed model is $1.04 \%$, essentially all of which comes from the neglected change in energy stored in the refrigerant. The errors in Eq. (21) for the Quasi-Transient and the Mapped-Component models were also evaluated, and were found to be $3.37 \%$ for the Quasi-Transient model which is reasonable. For the Mapped-Component model, the error was $1.1 \%$, which is very good but is most likely a result of the cancellation of multiple inaccuracies.

\section{Model Execution Speed}

These small losses of accuracy come at a large benefit to simulation execution speed. In Figure 13, the simulation execution speed is compared for the three model versions. The speeds were evaluated with the same simulation runs of the SC03 cycle that were shown in Figures 10 and $\underline{11}$. The simulation execution speed for the Fully-Detailed, QuasiTransient, and Mapped-Component models were 0.10, 0.98, and 9.96 times faster than real time respectively. For reference, "10 times faster than real time" speed means that one second of simulated time takes 0.1 second to simulate.

Execution speed is also dependent on the number of segments in the heat exchanger tubes or plates for the Fully-Detailed and the Quasi-Transient models. In both, for the results presented here, 18 segments for the condenser flat tubes and 10 segments for the evaporator plates were used. Fewer segments increase simulation speed due to both fewer operations per time step and the possibility of using a larger time step, but at a cost of reduced accuracy.

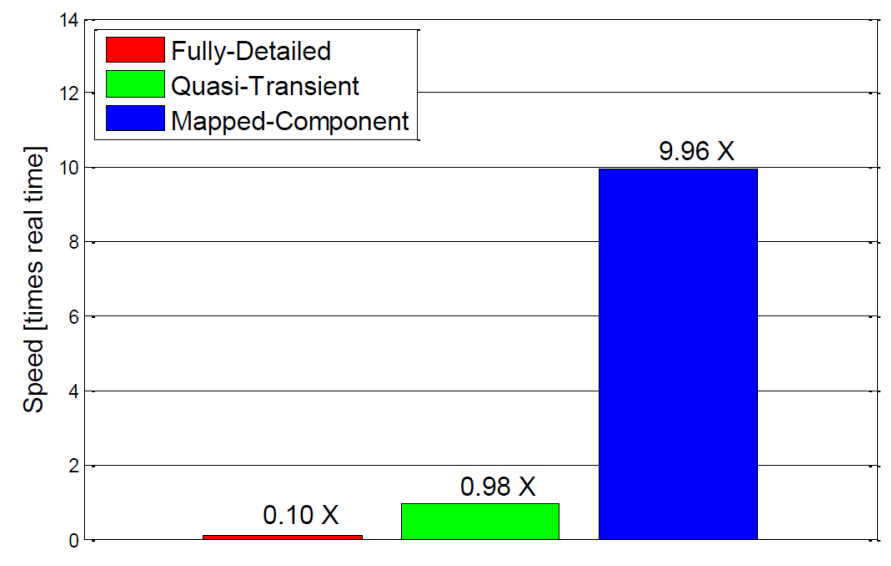

Figure 13. Comparison of model execution speeds

\section{CONCLUSIONS}

A new automotive $A / C$ system simulation tool was previously developed on the MATLAB/Simulink platform. That model consists of a detailed cooling circuit model and a relatively simple cabin model. For the key system simulation blocks, a finite volume formulation of the governing equations was used on the refrigerant side, which provided a very accurate preservation of refrigerant mass and a very accurate energy balance. In theory, the model can predict the fast transients that occur in an automotive A/C system. However, there was a need for much faster models better suited for vehicle drivecycle based evaluation of $A / C$ systems.

Therefore, based on the original model, new model versions were created with the goal of greatly increasing the simulation speed while minimizing loss of accuracy. The new models include the Quasi-Transient model and the MappedComponent model. The Quasi-Transient model provides essentially the same accuracy for steady state condition prediction as the Fully-Detailed model.

The Mapped-Component model does lose some accuracy in steady state conditions but still performs within about $2 \%$ average error from the results of the Fully-Detailed model and within about $2.5 \%$ average error from measured data over a wide range of operating conditions.

The simplified models are not expected to be suitable for analyzing problems such as refrigerant redistribution or refrigerant peak pressure after compressor shutdown or startup.

Cycle-based averaged results for compressor power and heat exchange rates for the SC03 cycle obtained with the QuasiTransient model are within $1.5 \%$ of the results of the Fully- 
Detailed model. The Mapped-Component model results are within $4.0 \%$ of the results of the Fully-Detailed model except for the compressor power, which was $18 \%$ off.

The respective speeds at which the simplified Quasi-Transient and Mapped-Component models run are 0.98 and 9.96 times real time speed. This represents a large improvement from the Fully-Detailed model, which runs 0.10 times real time speed. Conversion from one $\mathrm{A} / \mathrm{C}$ system model approach to another of the three models (Fully-Transient, Quasi-Transient, and Mapped-Component) is relatively simple. This allows a new model to be developed in a faster version before the results are refined using a slower, more detailed solution method as needed.

In the trade-off of speed and accuracy, the three models occupy very different parts of the scale, and it is believed that the simplified models are significant additions to the suite of models, especially for vehicle-focused, cycle-based evaluation of $A / C$ systems.

\section{REFERENCES}

1. Rugh, J.P., Hoveland, V., and Andersen, S.O. "Significant Fuel Savings and Emission Reductions by Improving Vehicle Air Conditioning," Earth Technologies Forum/Mobile Air Conditioning Summit, 2004

2. Francfort J. and Murphy T., (2007). Ch. V. "Operational And Fleet Testing, A. Hybrid Electric Vehicle Testing," Advanced Vehicle Technology Analysis and Evaluation Activities, FY 2007 Annual Report. Vehicle Technologies Program, U.S. Department of Energy. p. 145

3. Noyama, H. and Umezu, K., "Air-Conditioning System for Electric Vehicles (i-MiEV)," Presented at SAE Automotive Refrigerant System Efficiency Symposium, July 2010.

4. Lohse-Busch, H., Duoba, M., and Meyer, M. Advanced Powertrain Research Facility AVTA Nissan Leaf Testing and Analysis. Presentation. Argonne National Lab., IL, October 12, 2012.

5. Gaines, L., Vyas, A., and Anderson, J., "Estimation of Fuel Use by Idling Commercial Trucks," 85th Annual Meeting of the Transportation Research Board, Washington, D.C., Paper No. 062567, January 22-26, 2006.

6. Autonomie, Computer Software, Argonne National Laboratory. Access date: January $8^{\text {th }}, 2014$. www.autonomie.net

7. Hemami, T.L., "Development of a Transient System Model of Mobile Air-Conditioning Systems," ACRC Technical Report - 143, September 1998.

8. Cullimore, B.A., and Hendricks, T.J., "Design and Transient Simulation of Vehicle Air Conditioning Systems," VTMS 5 2001-011692, 2001.

9. Anand, G., Mahajan, M., Jain, N., Maniam, B. et al., "e- Thermal: Automobile Air-Conditioning Module," SAE Technical Paper 200401-1509, 2004, doi:10.4271/2004-01-1509.

10. Kiss, T., Chaney, L., and Meyer, J., "A New Automotive Air Conditioning System Simulation Tool Developed in MATLAB/ Simulink," SAE Int. J. Passeng. Cars - Mech. Syst. 6(2):826-840, 2013, doi:10.4271/2013-01-0850.

11. Streeter, V.L., and Wylie, E.B., "Fluid Mechanics" 7th edition, McGraw-Hill, New York, ISBN 0-07-062232-9, 1979.

12. Chang, Y., and Wang, C., "A Generalized Heat Transfer Correlation for Louver Fin Geometry," Int. J. Heat and Mass Transfer, 40(3):533-544, 1997.

13. Incropera, F.,P., and DeWitt, D.P., "Fundamentals of Heat and Mass Transfer," 2nd edition, John Wiley and Sons, Inc., New York, ISBN 0-471-88550-9, 1985.

14. Chen, J.C., "Correlation for Boiling Heat Transfer to Saturated Fluids in Convective Flow," I\&EC Process Design and Development 5(3):322-329, 1966.

15. Kandlikar, S.G., "Fundamental Issues Related to Flow Boiling in
Minichannels and Microchannels," Exp. Thermal Fluid Sci. 26:389407, 2002.

16. Zhang, W., Hibiki, T., and Mishima, K., "Correlation for Flow Boiling Heat Transfer in Mini-Channels," Int. J. Heat and Mass Transfer 47:5749-5763, 2004

17. Grosse, G., Robidou, H., and Chevallier, C., "Heat Transfer in Minichannels Tubes for Automotive Evaporator," SAE Technical Paper 2006-01-1449, 2006, doi:10.4271/2006-01-1449.

18. Cengel, Y.A., and Boles, M.A., "Thermodynamics - An Engineering Approach," McGraw-Hill, New York, ISBN 0-07-113249-X, 1994.

\section{CONTACT INFORMATION}

Tibor Kiss

tibor.kiss@nrel.gov

Jason Lustbader

jason.lustbader@nrel.gov)

\section{ACKNOWLEDGMENTS}

The authors would like to thank John Rugh and Lawrence Chaney of NREL, and John Meyer of Halla Visteon Climate Control for their contribution to this project.

The authors would also like to thank David Anderson and Lee Slezak, Technology Managers for the U.S. Department of Energy's Advanced Vehicle Technology Analysis and Evaluation for sponsoring this work.

\section{DEFINITIONS/ABBREVIATIONS}

0-D - zero-dimensional

1-D - one-dimensional

A/C - air conditioning

E-NTU - Effectiveness-Number of Transfer Units method

\section{Nomenclature}

A - Area for heat transfer

$\boldsymbol{\alpha}$ - Local heat transfer coefficient

B - Bulk modulus

C - Pipe flow convergence coefficient

$\boldsymbol{C}_{\boldsymbol{p}}$ - Constant pressure specific heat

$D_{H}$ - Hydraulic diameter

$\boldsymbol{f}$ - Pipe friction factor

$\boldsymbol{H}$ - Enthalpy

$\dot{H}$ - Enthalpy flow rate

$\boldsymbol{h}$ - Specific enthalpy

$L$ - Length of pipe or pipe segment

$\boldsymbol{m}$ - mass

$\dot{m}$ - Refrigerant mass flow rate

$\boldsymbol{P}$ - Power

p - Pressure

$\dot{Q}$ - Heat transfer rate

$\dot{m}$ - Refrigerant mass flow rate

$\boldsymbol{\rho}$ - Density 
$\rho_{\text {ref }}$ - Reference or 'zero-pressure' density

$T$ - Temperature

$\boldsymbol{t}$ - Time

$\boldsymbol{U}$ - Internal energy

$\boldsymbol{V}$ - Volume

$\boldsymbol{v}$ - Flow velocity

$\boldsymbol{w}$ - Flow channel perimeter

$\boldsymbol{x}$ - Coordinate in direction of flow

$\boldsymbol{y}$ - Segment length portion

\section{Subscripts}
a - Artificial
air - Air flow related
ave - Time averaged
aw - Air to wall
compr - Compressor
cond - Condenser
d - Downstream
evap - Evaporator
in - On inflow boundary
I - Liquid
out - On outflow boundary
sat - Saturation property
$\boldsymbol{u}$ - Upstream
V - Vapor
$w$ - Wall
$w r$ - Wall to refrigerant

\footnotetext{
This is a work of a Government and is not subject to copyright protection. Foreign copyrights may apply. The Government under which this paper was written assumes no liability or responsibility for the contents of this paper or the use of this paper, nor is it endorsing any manufacturers, products, or services cited herein and any trade name that may appear in the paper has been included only because it is essential to the contents of the paper.

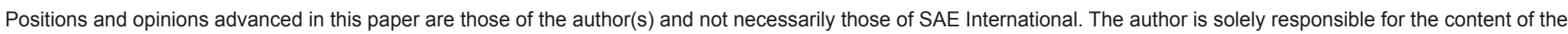
paper.
} 